\title{
Estudio de aplicación de modelos didácticos de Ciencias Experimentales en un proyecto comunidad de aprendizaje
}

\author{
Vanessa Ortega-Quevedo. Universidad de Valladolid \\ 0000-0002-5742-4678 \\ Cristina Gil Puente. Universidad de Valladolid \\ 0000-0001-5794-5564
}

Recepción: 14.11.2018| Aceptado: 30.01.2019

Correspondencia a través de ORCID: Vanessa Ortega Quevedo

0000-0002-5742-4678

Citar: Ortega-Quevedo, V. y Gil, C. (2018). Estudio de aplicación de modelos didácticos de Ciencias Experimentales en un proyecto Comunidad de Aprendizaje. ReiDoCrea, 8, 80-94.

\begin{abstract}
Resumen: El objetivo principal de este estudio es realizar un diagnóstico sobre el uso de modelos didácticos propios de la enseñanza de las ciencias por parte de los docentes de una Comunidad de Aprendizaje, además de observar cómo afecta la inclusión de una concepción comunicativa de la enseñanza en la aplicación de las distintas estrategias de enseñanza-aprendizaje propias de las Ciencias Experimentales. Los instrumentos de recogida de información empleados son un cuestionario aplicado a los docentes y las reflexiones del diario de clase. Como resultado se observa que los docentes del centro Comunidad de Aprendizaje estudiado no implementan estrategias de enseñanza-aprendizaje comunes a un solo modelo didáctico, sino que las combinan; así como que la integración de la concepción comunicativa de la enseñanza en la implementación de estrategias de enseñanza propias de las Ciencias Experimentales favorece la motivación y la reflexión profunda, lo cual crea un ambiente propicio para el desarrollo de la alfabetización científica del alumnado.
\end{abstract}

Palabras clave: Educación Básica | Enseñanza de las Ciencias

Study of didactic models employed in the Experimental Sciences. Implementation in a learning community project

\begin{abstract}
The main objective of this project was to make a diagnosis about the use of teaching models specific to the teaching of science by teachers of a learning community, in addition to observing how it affects the inclusion of a communicative conception of teaching in the application of the different teachinglearning strategies of the experimental sciences. The instruments used for the collection of information were a questionnaire that was completed by teachers and the reflections of the class diary. The results showed that the teachers of the community learning school studied do not implement teaching-learning strategies common to a single didactic model but rather they combine them; as well as that the integration of the communicative conception of teaching in the implementation of experimental science teaching strategies favours motivation and deep reflection, which creates an environment conducive to the development of students' scientific literacy.
\end{abstract}

Keywords: Elementary Education | Science Teaching

\section{Introducción}

"La ciencia y la tecnología tienen una presencia ubicua, notoria y significativa en la sociedad. Sus repercusiones en la economía, la política, la educación, la cultura, el ocio, etc." (Vázquez y Manassero, 2012, p.1), hacen que su comprensión sea trascendental a la hora de tomar decisiones en la sociedad actual. De lo que se deduce que la población necesita poseer una serie de habilidades en Ciencia y Tecnología (СуT), para poder ejercer plenamente su ciudadanía en la sociedad. De ahí que sea necesario plantear una nueva forma de entender la enseñanza de las ciencias que esté en consonancia con la nueva realidad social y que permita entender esta de una forma inclusiva. Para ello se ha de tener en cuenta que no solo se están educando futuros científicos o ingenieros, sino también a miembros activos de una sociedad donde la CyT juegan un papel fundamental (Solbes, Montserrat y Furió, 
2007; Vázquez y Manassero, 2012; Pedrinaci, Caamaño, Cañal, y de Pro, 2012; Martín, Prieto, y Jiménez, 2015).

No obstante, la literatura científica muestra como en los últimos cursos de Educación Primaria y durante toda la Educación Secundaria se incrementa sucesivamente el desinterés por las materias científicas (Solbes et al., 2007; Vázquez y Manassero, 2011). En otras palabras, los educandos pierden de forma progresiva la curiosidad y la motivación por el aprendizaje de los contenidos trabajados, y se introducen en un proceso pasivo donde prima la desmotivación y el fracaso académico. Algunas de las causas de este fenómeno se exponen a continuación (Solbes, 2011; Solbes et al.,2007).

La valoración social negativa de la ciencia: la sociedad actual entiende las materias científicas como áreas de conocimiento muy complejas a las que pueden optar solo genios y las cuales trabajan en proyectos que en ocasiones resultan peligrosos para la sociedad o el medio ambiente (Dunbar, 1999). Asimismo, se ha llegado a concluir que la población actual, en general, cuenta con visiones inadecuadas sobre el saber científico (Pujalte, Bonan, Porro, y Adúriz-Bravo, 2014).

La enseñanza de las ciencias, generalmente, tiene un enfoque teórico y descontextualizado que desmotiva al alumnado (Solbes et al., 2007), mientras que un enfoque que enfatice otros aspectos, como el conocimiento sobre CyT, podría resultar atractivo y permitir que se aumente el número de estudiantes que realicen estudios científicos.

En relación con las causas del desinterés se observan una serie de dificultades asociadas al proceso de enseñanza-aprendizaje de las Ciencias Experimentales, como pueden ser (Torres, 1975; Solbes et al., 2007; Vílchez et al. 2015):

- Las materias de ciencias están orientadas a la consecución en masa de una serie de contenidos que no son contextualizados, es decir se entiende la ciencia como una materia enciclopédica.

- Los educandos reciben la información como una avalancha de datos que deben memorizar y retener, no como respuestas a preguntas.

- El uso desmedido de los libros de texto, como base y referencia en el proceso de enseñanza, pese a que, en ocasiones, la interpretación de los contenidos curriculares que estos recogen es incompleta o incorrecta.

- El escaso planteamiento práctico de los contenidos y las malas programaciones. Las experiencias prácticas deben estar contextualizadas y los discentes precisan conocer su finalidad y su relación con la teoría.

- La falta de una perspectiva histórica de la ciencia, así como el aislamiento de los contenidos científicos entre las distintas áreas, causan que los temas se traten de un modo inconexo, dificultando así su comprensión.

- La aplicación de una evaluación cuantitativa y de exámenes externos, procesos de evaluación dirigidos a calificar el contenido memorizado y no otras formas de aprendizaje.

- Las carencias en la formación del profesorado. Los docentes generalmente muestran un gran desconocimiento, tanto de los contenidos curriculares de Ciencias Experimentales, como del modo en el que deben enseñarlos.

Dichas dificultades pueden solventarse con una nueva interpretación de la enseñanza de las ciencias, como las que proponen algunos enfoques relativos a la NdCyT, Estos enfoques constituyen uno de los movimientos más innovadores que promueven el cambio en la enseñanza de las ciencias, para acabar con el desinterés y conseguir la 
alfabetización científica del alumnado (Vázquez, Manassero, Acevedo, Acevedo, 2007; Vázquez y Manassero, 2012).

En este contexto, el término alfabetización científica se define como la capacidad de una persona para: preguntar o hallar respuestas que satisfagan su curiosidad científica, para comprender artículos sobre ciencia y poder participar en debates sociales acerca de estos, para posicionarse con conocimiento ante una decisión política que afecte a un aspecto científico-tecnológico o para valorar la calidad de la información científica, entre otras cuestiones (Pedrinaci et al., 2012). Ahora bien, el desarrollo de las competencias científico-tecnológicas que promueven la alfabetización científica deben de ser accesibles para todas las personas independientemente de sus características o condiciones específicas. Sin embargo, parte de la población, en ocasiones, quedan alejados de este tipo de formación. Esto se debe a que hoy en día muchos centros educativos continúan organizándose en base a los principios educativos que se utilizaban en las escuelas creadas a partir de la Revolución Industrial. Esta concepción mantiene una percepción dualista de la enseñanza, ya que sitúa la excepcionalidad académica en los estudios universitarios y de bachillerato, y mantiene una educación elemental para quien no pueda alcanzar dichos niveles (DíezPalomar, y Flecha, 2010; Flecha, Prados, y Puigdellívol, 2003). No obstante, proyectos educativos como el denominado Comunidades de Aprendizaje proponen una forma alternativa de entender la educación (Elboj, Puigdellívol, Soler, y Valls, 2005). Una Comunidad de Aprendizaje sería entonces un proyecto que persigue el cambio social y cultural de un centro educativo y de su entorno. Este se basa en el aprendizaje dialógico y pretende ofrecer una educación en la que participe el conjunto de la comunidad. Dicha participación consiste en el trabajo de igual a igual entre familiares, docentes y voluntarios, con el fin último de facilitar igualdad de oportunidades para los discentes en la sociedad actual (Valls, 2005). Así pues, las Comunidades de Aprendizaje "se caracterizan por los roles y las reglas básicas que rigen la conducta de sus miembros y cómo depende el uso del lenguaje para el pensamiento colectivo en actividades compartidas de grupos establecidos con intereses y objetivos comunes" (de la Hoz, 2011, p.46).

En esta línea, el proceso de enseñanza-aprendizaje, entendido desde la perspectiva de los proyectos Comunidad de Aprendizaje, sufre un importante cambio al incorporar nuevas prácticas que potencian el desarrollo de habilidades creativas y de pensamiento. Habilidades que son fundamentales para que los educandos se formen y puedan ejercer su ciudadanía en el nuevo escenario social actual (de la Hoz, 2011). Por lo tanto, el proceso de transformación de un centro en Comunidad de Aprendizaje se consigue mediante la implementación de 8 fases (Cifuentes y Fernández, 2010; Elboj et al., 2005) que pueden verse en la Figura 1.

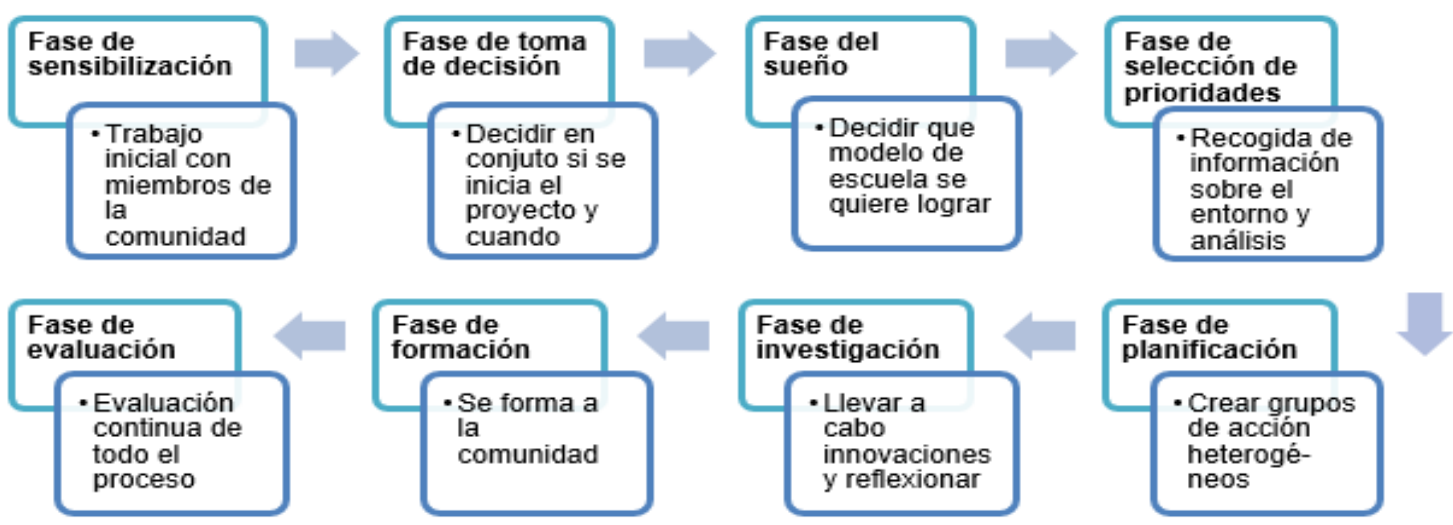

Figura 1. Fases de transformación de un colegio en Comunidad de Aprendizaje 
En cuanto a los modelos de enseñanza conviene resaltar que en la enseñanza de las ciencias resulta enriquecedor dejar a un lado la concepción de los modelos de enseñanza estancos, para dejar paso a la implementación equilibrada de estrategias propias de distintos modelos (Garrido, Perales, y Galdón, 2007). Con el fin de identificar las distintas estrategias propias de los diferentes modelos didácticos se recopilan las principales características de estos (véase Tabla 1). Del mismo modo, es preciso destacar que los recursos didácticos son una parte fundamental del proceso de enseñanza-aprendizaje y, en ocasiones, la falta de ellos origina clases magistrales (García-Ruiz y Orozco, 2008). Por tanto, es preciso contemplar una amplia variedad de recursos didácticos dentro de las programaciones (maquetas, audiovisuales, juegos, etc.).

Tabla 1. Resumen de los diferentes modelos didácticos

\begin{tabular}{|c|c|c|c|c|c|c|c|c|}
\hline & \multicolumn{2}{|c|}{ Fundamentos } & \multirow{2}{*}{ Principios } & \multirow{2}{*}{$\begin{array}{l}\text { Modelo en } \\
\text { acción }\end{array}$} & \multirow{2}{*}{ Docente } & \multirow{2}{*}{ Discente } & \multirow{2}{*}{$\begin{array}{l}\text { Organización } \\
\text { del aula }\end{array}$} & \multirow{2}{*}{ Recursos } \\
\hline & psicológicos & epistemológicos & & & & & & \\
\hline $\begin{array}{l}\text { 总 } \\
\text { 总 } \\
\text { 总 } \\
\text { 总 } \\
\text { 总 }\end{array}$ & $\begin{array}{l}\text { Alumno es un } \\
\text { recipiente } \\
\text { vacio. }\end{array}$ & $\begin{array}{l}\text { Concepción de } \\
\text { la ciencia } \\
\text { estática. }\end{array}$ & $\begin{array}{l}\text { Reproducci- } \\
\text { ón exacta de } \\
\text { los } \\
\text { contenidos } \\
\text { cientificos. }\end{array}$ & $\begin{array}{l}1^{\circ} \text { lección } \\
\text { magistral } \\
2^{\circ} \text { ejercicios } \\
\text { individuales. }\end{array}$ & $\begin{array}{l}\text { Transmisor } \\
\text { de conoci- } \\
\text { miento. }\end{array}$ & $\begin{array}{l}\text { Escasa } \\
\text { participaci- } \\
\text { ón. }\end{array}$ & $\begin{array}{l}\text { Gran grupo } \\
\text { con } \\
\text { orientación a } \\
\text { la pizarra. }\end{array}$ & $\begin{array}{l}\text { Libro de } \\
\text { texto. }\end{array}$ \\
\hline 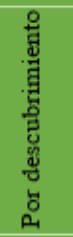 & $\begin{array}{l}\text { Los niños } \\
\text { aprenden } \\
\text { cuando } \\
\text { descubren. }\end{array}$ & $\begin{array}{l}\text { E1 empirismo e } \\
\text { inductismo. }\end{array}$ & $\begin{array}{l}\text { Llegar al } \\
\text { conocimien- } \\
\text { to a partir de } \\
\text { procesos. }\end{array}$ & $\begin{array}{l}\text { Enfrentar a los } \\
\text { alumnos a } \\
\text { problemas. }\end{array}$ & $\begin{array}{l}\text { Creador de } \\
\text { guiones de } \\
\text { trabajo y } \\
\text { coordinador. }\end{array}$ & $\begin{array}{l}\text { Participació } \\
\text { n activa. }\end{array}$ & $\begin{array}{l}\text { No es } \\
\text { determinante, } \\
\text { pero ha de } \\
\text { estar en } \\
\text { concordancia } \\
\text { con el material } \\
\text { y la práctica. }\end{array}$ & $\begin{array}{l}\text { Labora- } \\
\text { torio. }\end{array}$ \\
\hline 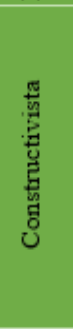 & $\begin{array}{l}\text { Construcción } \\
\text { personal del } \\
\text { conocimiento } \\
\text {, a partir de lo } \\
\text { que se } \\
\text { conoce. }\end{array}$ & $\begin{array}{l}\text { La ciencia } \\
\text { avanza } \\
\text { reconstruyendo } \\
\text { ideas cientificas } \\
\text { previas. }\end{array}$ & $\begin{array}{l}\text { Aprender es } \\
\text { reconstruir } \\
\text { ideas y } \\
\text { conceptos. }\end{array}$ & $\begin{array}{l}1^{\circ} \text { activación } \\
\text { de } \\
\text { conocimientos } \\
\text { previos. } \\
2^{\circ} \text { cuestiones } \\
\text { que permitan } \\
\text { reflexionar, } \\
\text { realizar } \\
\text { prácticas e } \\
\text { interactuar. }\end{array}$ & $\begin{array}{l}\text { Motivador, } \\
\text { guía, e } \\
\text { investigador } \\
\text { en el } \\
\text { proceso de } \\
\text { enseñanza- } \\
\text { aprendizaje. }\end{array}$ & $\begin{array}{l}\text { Responsabil } \\
\text { idad del } \\
\text { proceso de } \\
\text { aprendizaje. }\end{array}$ & $\begin{array}{l}\text { Flexible, } \\
\text { puede variar } \\
\text { entre } \\
\text { pequeños } \\
\text { grupos o gran } \\
\text { grupo. }\end{array}$ & $\begin{array}{l}\text { Escritos y } \\
\text { experi- } \\
\text { mentales. }\end{array}$ \\
\hline
\end{tabular}

Nota. Fuente: elaboración basada en Vílchez (2015) y Garrido et al. (2007).

En lo relativo a los proyectos Comunidad de Aprendizaje se destaca que estos han adoptado las teorías dialógicas de la enseñanza basándose en los cambios sociales y en las teorías presentadas por diversos autores (Vygotsky, 1996; Bruner, 2000; Freire, 1970), quienes estiman que el diálogo y la interacción de los discentes, no solo entre ellos, sino también con las personas de su entorno, incrementan el aprendizaje y favorecen el desarrollo personal y cultural. En concreto se determina que el aprendizaje dialógico es aquel "que resulta de las interacciones que produce el diálogo igualitario, es decir, un diálogo en el que diferentes personas aportamos argumentos en condiciones de igualdad" de esta forma se origina un proceso de creación del conocimiento en el que cada persona aporta sus saberes, sentimientos y experiencias (Aubert, Flecha, García, Flecha y Racionero, 2010).

Una de las aplicaciones más representativas del aprendizaje dialógico se ve reflejada en los grupos interactivos, pues a través de esta actividad los discentes interactúan con sus compañeros y con los voluntarios presentes en el aula, enriqueciendo de esta forma su aprendizaje. Con esta aportación, se intuye que los grupos interactivos son una estrategia didáctica inspirada en una concepción comunicativa de la educación. Sin embargo, estos son una forma de organización del aula que consiste en la división 
de la clase en pequeños grupos con el objetivo de promover el éxito educativo de todos.

\section{Objetivos}

Los objetivos específicos establecidos para esta investigación consisten en: (1) realizar un diagnóstico sobre el uso de modelos didácticos propios de la enseñanza de las ciencias por parte de los docentes de una Comunidad de Aprendizaje. (2) Diseñar e implementar una propuesta didáctica que integre distintas estrategias de enseñanza de las ciencias con metodologías propias del aprendizaje dialógico para promover el desarrollo de una alfabetización científica mediante el abordaje de temáticas sobre Ciencia Tecnología y Sociedad (CTS).

\section{Métodos}

Se plantea un estudio de carácter exploratorio que permita conocer cómo se afronta la enseñanza de las ciencias en un centro Comunidad de Aprendizaje situado en Segovia (ciudad pequeña donde los centros escolares con estas características son un número muy reducido. El diseño de este estudio se divide en dos partes. Un parte se centra en esclarecer que estrategias de enseñanza de las ciencias emplean los docentes de Ciencias de la Naturaleza del centro; esta se abordará mediante una metodología cuantitativa y un análisis estadístico descriptivo y correlacional de los datos. Por otro lado, la segunda parte del estudio se centra en el diseño e implementación de una propuesta didáctica que mediante distintas estrategias trabaja temáticas sobre CTS y promueve la alfabetización científica ciudadana. En esta segunda parte de la investigación se realiza un análisis reflexivo de los resultados de la implementación de la propuesta mediante los datos extraídos del diario de clase.

\section{Estudio diagnóstico sobre las estrategias didácticas implementadas por el profesorado}

Instrumento de recogida de datos

La recogida de datos cuantitativos se ha realizado mediante un cuestionario. En este instrumento se establecen categorías como: actitud hacia el área de Ciencias Naturales; criterios de selección, secuenciación y organización de contenidos en la enseñanza de las Ciencias Naturales; actividades, estrategias y recursos utilizados para la enseñanza-aprendizaje. El resultado final, es un cuestionario formado por 2 preguntas de tipo socio-demográfico y 17 preguntas cerradas formuladas con una escala tipo Likert clásica que se define del valor 1 (no/nada) al 5 (mucho).

\section{Muestra y contexto del estudio}

Los participantes del estudio se han seleccionados según el denominado muestreo por conveniencia. Dichos participantes son los docentes de Ciencias Naturales de un centro Comunidad de Aprendizaje situado en Segovia capital. El total de participantes se compone por 10 docentes, de los cuales 5 son mujeres y 5 hombres.

\section{Procedimiento de análisis de la información}

El tratamiento de la información recogida en esta primera parte del estudio se ha realizado en dos fases. Durante la primera fase se presenta la descripción 
de los datos recogidos y se relacionan con la teoría relevante. Por otra parte, en segunda fase los datos obtenidos se han tratado de forma estadística mediante el programa SPSS (20.0) con el fin de realizar un análisis correlacional, mediante la el coeficiente de correlación de Pearson, y responder a una serie de hipótesis sobre las relaciones entre las variables descritas en la primera fase.

\section{Estudio sobre la propuesta didáctica}

\section{Instrumento de intervención didáctica}

La programación se ha diseñado de forma que se implementen distintas estrategias didácticas para enriquecer el proceso de enseñanza-aprendizaje, asimismo se realiza un tratamiento transversal durante todas las sesiones para trabajar temas sobre CTS tales como: influencia de la ciencia y la tecnología en la sociedad, y construcción social de la tecnología. La Tabla 2 recoge un esquema de los contenidos y estrategias aplicados en dicha programación.

Tabla 2. Esquema de contenidos y estrategias implementados

\begin{tabular}{|c|c|c|}
\hline Sesiones & Contenido curricular & Estrategias didácticas \\
\hline Sesión 1 & $\begin{array}{l}\text { Concepto de Energía, propiedades y manifestaciones de la } \\
\text { energía }\end{array}$ & $\begin{array}{l}\text { Conocimientos previos } \\
\text { Debate } \\
\text { Diálogo (presente en todas las sesiones) } \\
\text { Evaluación formativa (presente en todas las } \\
\text { sesiones) }\end{array}$ \\
\hline Sesión 2 & Propiedades y manifestaciones de la energía & $\begin{array}{l}\text { Manipulación } \\
\text { Descubrimiento }\end{array}$ \\
\hline Sesión 3 & Propiedades y manifestaciones de la energía & $\begin{array}{l}\text { Método científico Experimentación } \\
\text { Grupos interactivos } \\
\text { Dispositivos electrónicos }\end{array}$ \\
\hline Sesión 4 & Fuentes de energía renovables y no renovables & $\begin{array}{l}\text { Conocimientos previos } \\
\text { Pensamiento Crítico } \\
\text { Descubrimiento } \\
\text { Debate }\end{array}$ \\
\hline Sesión 5 & Fuentes de energía renovables y no renovables & $\begin{array}{l}\text { Conocimientos previos } \\
\text { Pensamiento Crítico } \\
\text { Dispositivos electrónicos } \\
\text { Debate }\end{array}$ \\
\hline Sesión 6 & Todos los contenidos anteriores & $\begin{array}{l}\text { Pensamiento Crítico } \\
\text { Grupos interactivos } \\
\text { Experimentación }\end{array}$ \\
\hline Sesión 7 & Impactos ambientales relacionados con la energía & $\begin{array}{l}\text { Conocimientos previos } \\
\text { Pensamiento Crítico } \\
\text { Grupos interactivos } \\
\text { Debate }\end{array}$ \\
\hline Sesión 8 & Sostenibilidad & $\begin{array}{l}\text { Descubrimiento } \\
\text { Manipulación } \\
\text { Debate }\end{array}$ \\
\hline Sesión 9 & Todos los contenidos anteriores & $\begin{array}{l}\text { Pensamiento Crítico } \\
\text { Dispositivos electrónicos } \\
\text { Manipulación }\end{array}$ \\
\hline Sesión 10 & Todos los contenidos anteriores & Juego \\
\hline Sesión 11 & Todos los contenidos anteriores & Evaluación final \\
\hline
\end{tabular}

Instrumento de recogida de datos

El instrumento seleccionado para recoger los datos de la observación docente realizada durante la implementación de la intervención didáctica es el diario de clase. En concreto se ha empleado una tipología de diario denominada analytical writing (Zabalza, 2004), puesto que se quiere emplear este para 
analizar aspectos concretos dentro de un determinado contexto de observación.

\section{Muestra y contexto de intervención}

Los estudiantes que han participado en la intervención didáctica pertenecen a un grupo de $5^{\circ}$ curso del centro Comunidad de Aprendizaje en el cual se realiza el estudio. Dicho grupo está conformado por un total de 20 estudiantes, de los cuales 8 son chicas y 10 chicos. Cabe destacar que 2 de los discentes se han incorporado al grupo a mitad del segundo trimestre.

\section{Procedimiento de análisis de la información}

El análisis de las reflexiones recogidas en el diario de clase se realizará de forma cualitativa, mediante la elaboración de categorías (Kvale, 2011). Las categorías se han determinado en base a las tres etapas definidas por Taylor y Bogdan (2010) identificar los temas, recoger y codificar la información y comprensión de los datos en base al concepto -. Dichas categorías son:

- Rutinas de pensamiento

- El uso de elementos audiovisuales y dispositivos electrónicos

- Manipulación y experimentación

- La contextualización de la teoría en la realidad

- Los debates y coloquios (reflexión e inmersión)

- El juego como recurso didáctico

\section{Resultados y discusión}

Con el fin de realizar un diagnóstico completo de la visión de la enseñanza de las ciencias de los participantes se va a realizar un análisis descriptivo de las respuestas obtenidas en el cuestionario, seguido de un análisis correlacional. En la Tabla 3 se recogen los resultados extraídos del cuestionario.

\section{Tabla 3. Resultados descriptivos del estudio diagnóstico}

1. ¿Tu actitud ante el área de Ciencias Naturales suele ser positiva?

El $60 \%$ de los docentes afirman mantener una actitud muy positiva hacia la materia seguidos por el $30 \%$ que manifiesta que su actitud es bastante positiva y quedando un $10 \%$ que muestra tener una actitud algo positiva.

2. ¿Tratas de resaltar la importancia de la aplicación en la vida real de los contenidos de Ciencias de la Naturaleza?

El $70 \%$ de los participantes manifiesta resaltar mucho la importancia de la aplicación de los contenidos en la vida real y el 30\% restante afirma resaltarlo bastante.

3. ¿Sueles plantear problemas como introducción a determinados contenidos del área?

El $60 \%$ de los maestros sostiene que emplean esta estrategia didáctica mucho o bastante, mientras que el $40 \%$ la emplean algo o poco.

4. ¿Tratas de partir de los conocimientos previos del alumnado a la hora de explicar los contenidos curriculares?

El $80 \%$ de los docentes manifiestan partir de los conocimientos previos del alumnado mucho, seguidos del $20 \%$ que indica hacerlo bastante.

5. ¿Propones actividades en las que el alumnado tenga que descubrir y comprender por sí mismo una serie de contenidos del currículo de Ciencias de la Naturaleza?

El $50 \%$ de los participantes sostiene que emplean estrategias de aprendizaje por descubrimiento algo, el $40 \%$ poco y el $10 \%$ nada.

6. ¿Utilizas libros de texto como apoyo para la enseñanza en el área?

El $20 \%$ de los docentes afirma apoyarse mucho en el libro de texto, el $70 \%$ bastante y el $10 \%$ algo.

7. ¿Intentas plantear ejercicios que fomenten el pensamiento crítico y la reflexión sobre los contenidos propios del área?

El $40 \%$ de los maestros admiten plantear muchos ejercicios que promueven el pensamiento crítico y la reflexión, el $50 \%$ bastante y el $10 \%$ algo.

8. ¿Propones debates que permitan discutir temas relacionados con la asignatura?

El $30 \%$ de los participantes manifiestan realizar muchos debates sobre temáticas del área, el $50 \%$ bastantes y el $20 \%$ algunos. 


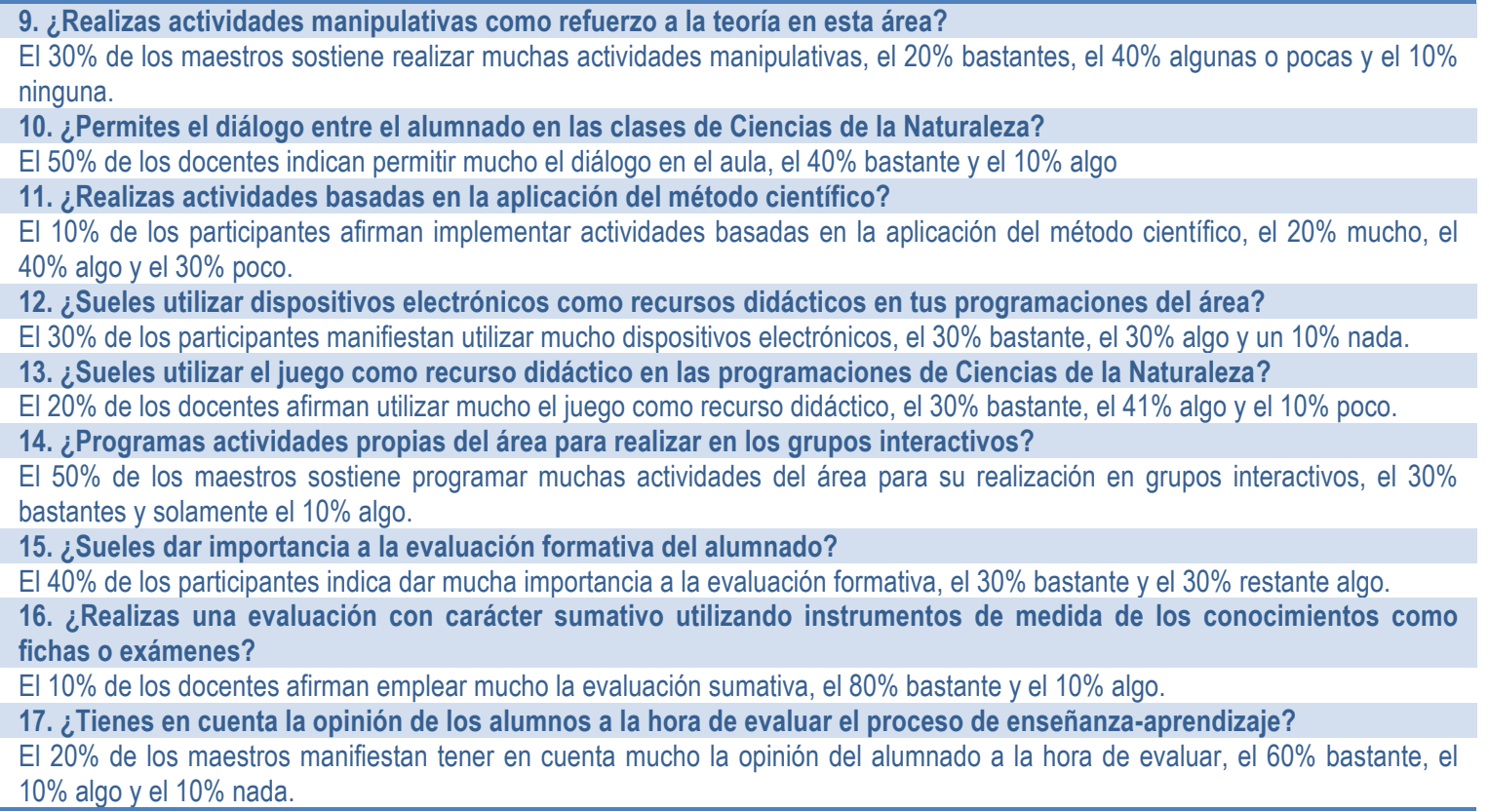

Tras esta presentación de los datos recogidos se observa que las estrategias relativas al modelo de aprendizaje por descubrimiento (el planteamiento de problemas, permitir que el alumnado descubra por sí mismo los conceptos y el uso del método científico en el aula) no se aplican de un modo usual en las programaciones. No obstante, aquellos docentes que suelen hacerlo tienden a tener más experiencia.

Durante el análisis se comprueba que el uso del modelo constructivista es concluyente, pues la mayor parte de los maestros manifiesta trabajar partiendo de los conocimientos previos del alumnado, teniendo muy presente la importancia de la contextualización de la teoría y promoviendo tanto el pensamiento crítico, como la reflexión. El uso combinado de estas estrategias, pueden situarse en el empleo de un estilo de enseñanza cognitivo con carácter funcional y con notas de un planteamiento abierto entendidos conforme a los expuesto por Renés (2018). Es decir, el docente estima el valor de los conocimientos previos para la reconstrucción de los esquemas cognitivos que preceden al aprendizaje, pone énfasis en la funcionalidad y la concreción de los contenidos en sus programaciones, prioriza los ejemplos y trabajos prácticos a las exposiciones magistrales, motiva a los dicentes mediante la implementación de actividades novedosas con problemas reales extraídos del entorno y fomenta la reflexión crítica.

Asimismo, se observa que el modelo de transmisión-recepción se mantiene presente en algunos aspectos como puede ser el uso de los libros de texto, que mantiene de forma activa una parte importante del profesorado, y el rechazo de las actividades manipulativas por el $50 \%$ del mismo. La combinación de estos dos estilos de enseñanza puede relacionarse con la corriente de profesionales de la enseñanza que defiende el uso del libro de texto como desarrollo del currículo y condicionan su actividad de enseñanza-aprendizaje en el seguimiento de dichos materiales (González, 2005). Sin embargo, es preciso resaltar que el uso que los docentes hagan de los libros de texto en su contexto de aula condiciona que estos sean utilizados como recursos no condicionantes del aprendizaje o como base del mismo (González, 2005).

Como último modelo didáctico investigado se encuentra el comunicativo. En este caso, a pesar de que el centro está inmerso en un proyecto Comunidad de Aprendizaje, 
existen docentes que no manifiestan utilizar debates y permitir el diálogo de forma usual. De esto se puede interpretar la reticencia de algunos docentes por estas dos estrategias didácticas basadas en la comunicación. En términos generales, aquellos que emplean menos estas estrategias metodológicas son los docentes con menor experiencia. Por otra parte, se observa que la mayor parte de los participantes y, en especial aquellos con más experiencia, programan actividades relacionadas con las Ciencias Naturales en las sesiones de grupos interactivos. Investigaciones como la planteada por Lavín (2014) permiten ver como la programación de actividades propias de las ciencias naturales mediante la implementación de esta estrategia didáctica promueve el desarrollo de habilidades propias del pensamiento científico y de habilidades cognitivas de orden superior que permiten la relación entre contenido y realidad natural y cultural; así como el aumento de la motivación.

Por otra parte, para establecer el análisis correlacional de las variables de estudio se determinan las siguientes hipótesis en base al contexto teórico. Así pues, tomando como referencia la fundamentación teoría basada en la aplicación de modelos didácticos estancos (como los presentados en la tabla 1 y a los que cabe añadir un modelo con aquellas estrategias propias de los proyectos Comunidad de Aprendizaje) se establece $\mathrm{H} 1_{0}$ y en base a la teoría presentada por Garrido et al. (2007) quienes determinan que en enseñanza de las ciencias se debe apostar por la implementación equilibrada de estrategias propias de distintos modelos se determina $\mathrm{H}_{1}$

- $\quad \mathrm{H} 1_{0}$ : Las correlaciones más fuertes se dan entre variables pertenecientes a los mismos modelos didácticos.

- $\mathrm{H} 1_{1}$ : Las correlaciones más fuertes no se dan únicamente entre variables pertenecientes a los mismos modelos didácticos.

Los resultados del análisis correlacional se representan en la Figura 2. En esta imagen se observa como cada una de las variables de estudio relativas a los modelos didácticos se representa en un rectángulo. En caso de existir correlaciones estas se representan con una flecha bidireccional que parte de un rectángulo con un color determinado y llegan a aquellas variables con las que están correlacionadas. Las correlaciones fuertes se representan mediante flechas más gruesas, mientras que las débiles con las más finas.

En base a los resultados expuestos en la Figura 2 se concluye que las correlaciones más fuertes no se dan únicamente entre variables pertenecientes a los mismos modelos didácticos y por lo tanto $\mathrm{H} 1_{1}$ queda comprobada. En otras palabras, los participantes implementan distintas estrategias con el fin de procurar una mejora de la enseñanza de las ciencias como proponen Garrido et al. (2007). Además, las correlaciones son positivas, lo cual indica que el uso de las estrategias correlacionadas aumenta o disminuye en conjunto.

Finalmente, tras el análisis de los resultados descriptivos se cree interesante comprobar qué variables de estudio están relacionadas con la experiencia docente. A priori parece que las estrategias didácticas consideradas comúnmente como más complejas (el planteamiento de problemas, el descubrimiento de contenidos por parte del alumnado, el trabajo a partir de métodos científicos, el fomento del diálogo y el debate, etc.) tienden a ser aplicadas por aquellos docentes con más experiencia.

- $\mathrm{H}_{2}$ : Existen correlaciones entre la experiencia docente y las variables que representan estrategias didácticas comúnmente adaptadas como complejas.

- $\mathrm{H} 2_{1}$ : No existen correlaciones entre la experiencia docente y las variables que representan estrategias didácticas comúnmente adaptadas como complejas. 
Los resultados del análisis correlacional se reflejan en la Figura 3. Estos resultados permiten observar la existencia de correlaciones positivas entre las variables presentadas en la Figura. Dichas correlaciones evidencian como el uso de las estrategias señaladas se incrementa o disminuye al mismo tiempo que la experiencia docente.

En base a los resultados, se confirma la relación entre el incremento de la experiencia y el aumento del uso de estrategias didácticas consideradas comúnmente como más complejas, muchas de estas fuertemente relacionadas con el modelo de aprendizaje por descubrimiento. Además, se advierte una correlación significativa con estrategias propias del constructivismo (aplicación en la vida real de los contenidos) orientado a un estilo de enseñanza abierto comúnmente relacionado con un estilo de aprendizaje activo (Renés, 2018); así como con el uso del juego como recurso didáctico.

A priori, estas evidencias pueden llevar a pensar en la experiencia como factor clave para que los docentes mejoren su confianza y su habilidad para implementar ciertas estrategias didácticas en el área de Ciencias de la Naturaleza. Una percepción que se ve reforzada por la fuerte correlación obtenida entre experiencia docente y actitud positiva hacia las ciencias. La sinergia entre estas variables motiva el planteamiento de nuevas incógnitas ¿la mejora de la actitud hacia las ciencias conforme aumenta la experiencia se debe a factores como la confianza en las habilidades docentes, al conocimiento de los contenidos del área, al conocimiento didáctico del contenido?
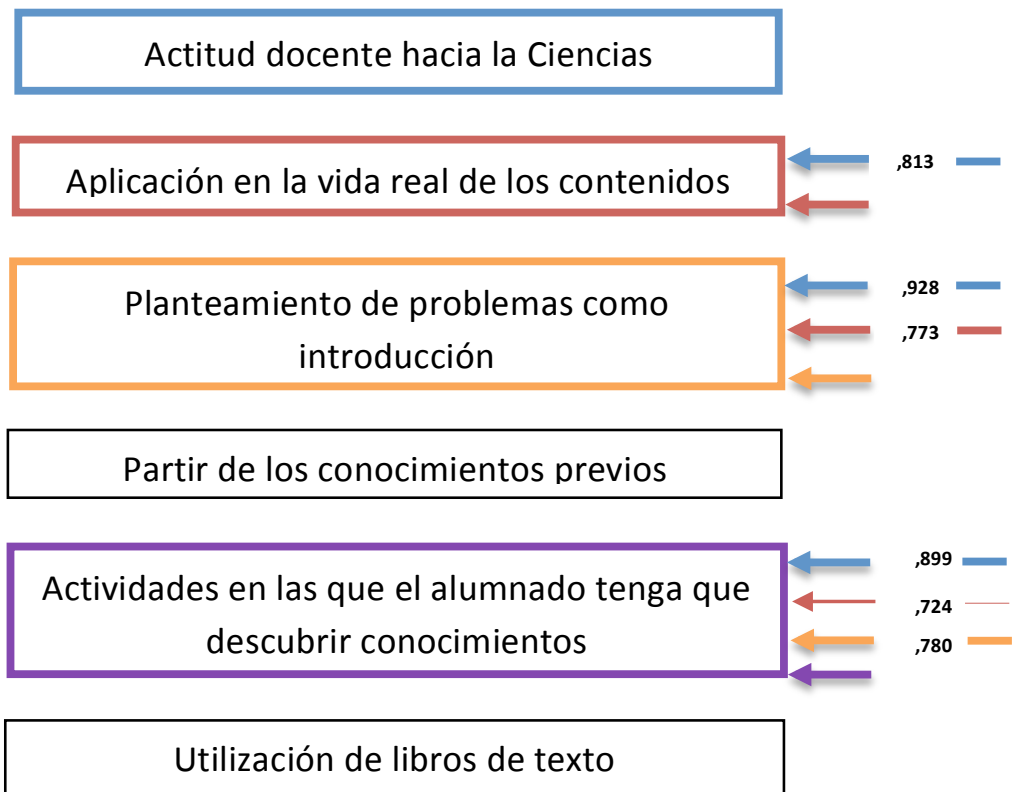

Actividades que fomenten el pensamiento crítico

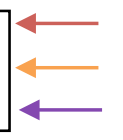
,648 , 643 , 659

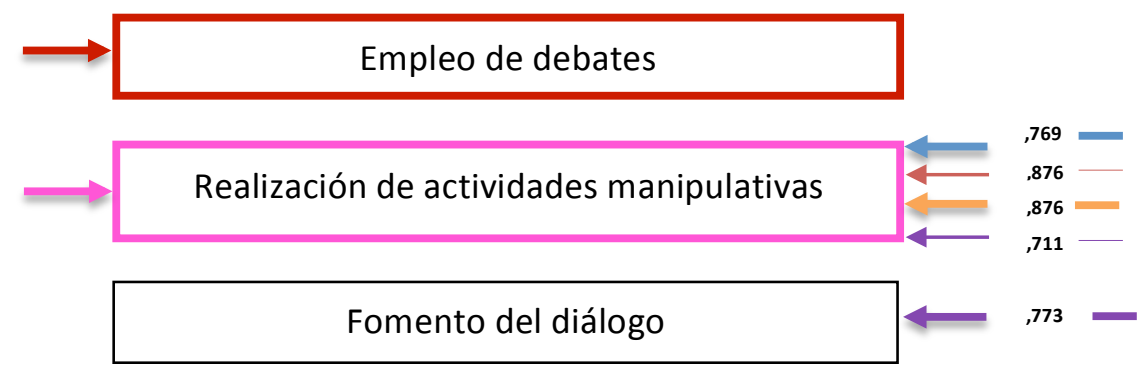




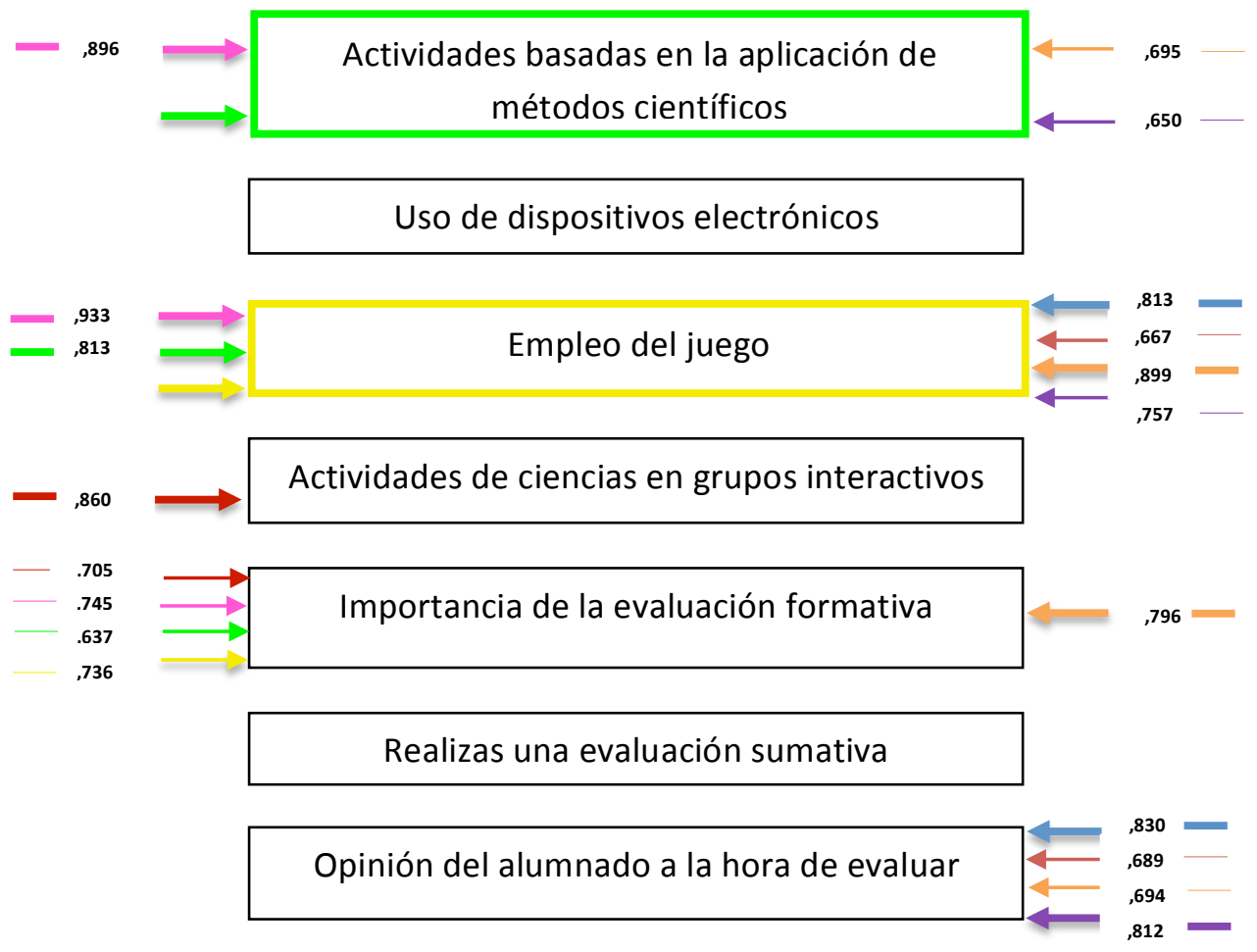

Figura 2. Resultados del análisis correlacional de las variables de los modelos didácticos
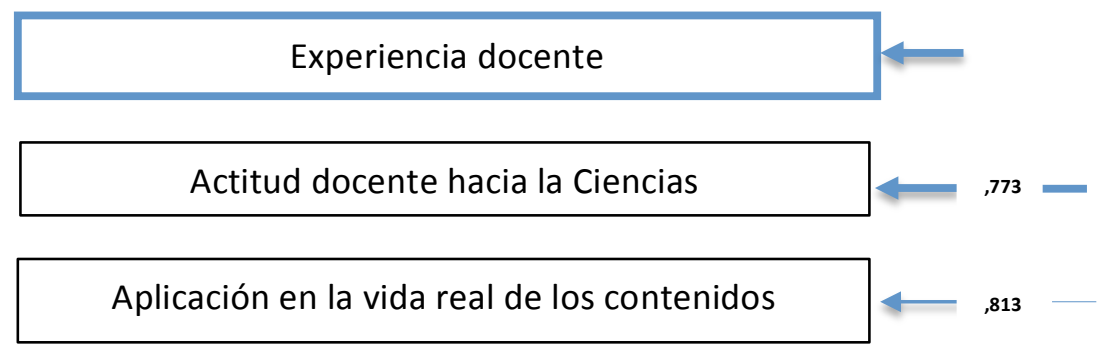

Planteamiento de problemas como introducción
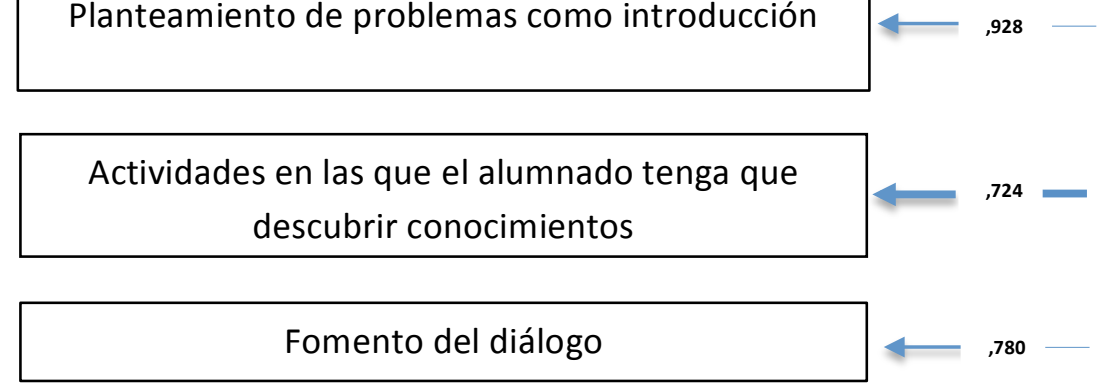

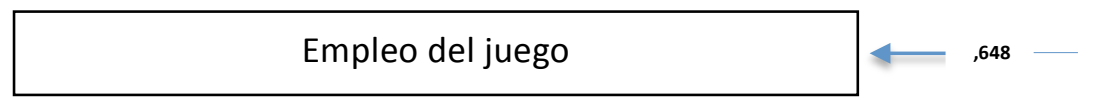

Opinión del alumnado a la hora de evaluar

Figura 3. Resultados del análisis correlacional de la variable experiencia docente 


\section{Estudio didáctico}

En el presente epígrafe se pasa realizar el análisis de las reflexiones plasmadas en el diario de clase sobre la intervención didáctica, en base a las categorías de análisis establecidas (rutinas de pensamiento; uso de elementos audiovisuales y dispositivos electrónicos; manipulación y experimentación; la contextualización de la teoría en la realidad; los debates y coloquios (reflexión e inmersión); y el juego como recurso didáctico). Sin embargo, antes de iniciar dicho análisis se cree preciso destacar que la totalidad de las actividades planteadas se han implementado sin incidencias destacables y no han tenido que tomarse medidas de replanteamiento.

\section{Rutinas de pensamiento}

A lo largo de la programación se han utilizado dos rutinas de pensamiento diferentes: veo, pienso, me pregunto y pensar, conectar, explorar. La primera rutina permite partir de la observación detallada de los estudiantes con el fin de profundizar en las ideas subyacentes a los elementos observados; mientras que la segunda permite cuestionar los saberes iniciales e indagar para ampliar dichos saberes (Ritchart, Church y Morrison, 2011). El uso de estas rutinas de pensamiento ha permitido: (1) hacer visible el pensamiento del alumnado (conocimientos previos) y que la maestra pudiera observar de que punto exacto partían los conocimientos de cada alumno, lo cual considera determinante para la implementación de las actividades sucesivas. (2) Incentivar la capacidad de reflexión de los discentes sobre una determinada temática y predisponerlos a modificar sus esquemas de conocimientos. (3) mejorar habilidades propias del pensamiento científico tales como la observación y el planteamiento de preguntas (Romero y Pulido, 2015).

\section{Uso de elementos audiovisuales y dispositivos electrónicos}

Durante gran parte de las sesiones se han utilizado recursos, tales como: videos, gráficos, mapas, fotografías, etc. que han servido como apoyo a la teoría y como forma de contextualizar la misma en la realidad. El punto de vista del docente recogido en el diario es claro "estos materiales han servido de punto de apoyo a los discentes para iniciar por sí mismos reflexiones en cuanto al contenido, así como para elaborar su propio conocimiento". De este modo, queda comprobado lo expuesto por Vílchez et al. (2015), quien argumenta que este tipo de recursos son de uso obligatorio y que se debe aprovechar su aplicabilidad en el contexto escolar.

\section{Manipulación y experimentación}

Durante dos sesiones diferentes se plantean experimentos que permiten observar las manifestaciones de la energía y sus propiedades. Con la experimentación, se contextualiza la teoría y se emplean procesos propios de la metodología científica. Esto ha permitido que los estudiantes se acerquen más a los procesos de investigación aplicados en Ciencias Experimentales, así como que desarrollen destrezas cognitivas, el pensamiento formal y habilidades como la observación, la formulación de hipótesis, la manipulación experimental, la inferencia, etc. (Jiménez, 2000). El docente refleja en el diario que dichas sesiones fueron un éxito, ya que los educandos consiguieron realizar satisfactoriamente todos los experimentos y las deliberaciones, recogidas en sus "cuadernos de notas del científico", dejan ver tanto el conocimiento de la teoría, como la reflexión sobre la misma. 
La contextualización de la teoría no es una actividad en sí misma, sino una forma de plantear las actividades que se introduce de forma transversal en la programación. Este estilo de enseñanza es propio de un constructivismo abierto (Renés, 2018) orientado a que los estudiantes sitúen los problemas teóricos en entornos cercanos y concretos. Esta contextualización cercana fomenta la reflexión y facilita la generación de debates con argumentaciones críticas y espontaneas fruto de las inquietudes del alumnado. En el diario de clase el docente recoge ejemplos de generación de inquietudes y reconocimiento de elementos teóricos en el día a día tales como: el reconocimiento de elementos teóricos sobre la tecnología fracking en programas de noticias y la satisfacción de comprender su significado; o la inclusión del diseño de un colegio sostenible en un proyecto ajeno al área por iniciativa propia del alumnado. Asimismo, se cree preciso señalar que la transferencia del contenido teórico-práctico a la vida diaria es el resultado del desarrollo de competencias de carácter científico y de la alfabetización científica del alumnado y como esto ha sido motivado por la propia contextualización de dichos contenidos en la realidad de los discentes.

\section{Los debates y coloquios (reflexión e inmersión)}

Conforme lo expuesto en el diario de clase, las actividades programadas en la intervención están basadas en una concepción comunicativa de la enseñanza, lo cual hace que sea inevitable fomentar los momentos de diálogo en el aula. Dichos espacios de tiempo dedicados a hablar sobre una temática según las reflexiones de la docente "son tremendamente enriquecedores para el alumnado, ya que no solo les permite expresar sus dudas, sino también reflexionar críticamente, participar, reconstruir sus esquemas de conocimientos previos, aprender a respetar el turno de palabra, así como las ideas de sus compañeros...". Un buen debate aplicado al aula genera preguntas, reflexiones y controversias que incentivan la participación e incrementan el interés y la curiosidad del alumnado, lo cual motiva la resolución de dudas y la generación de aprendizajes (Mosquera, 2017). Asimismo, la docente reflexiona sobre la contribución del debate como forma de atención a la diversidad, haciendo alusión a como las intervenciones de cada alumno en particular se ajustan a su propio nivel, de manera que estos pueden ir progresando en la adquisición de los contendidos conforme evoluciona el debate, además de, como las intervenciones y argumentaciones de sus compañeros les ayudan a reconstruir sus propias concepciones.

\section{El juego como recurso didáctico}

El juego es "la forma natural del aprendizaje infantil y la actividad principal en la vida de los niños" (García-Ruiz y Orozco, 2008, p. 547). Esto ha generado que su implementación durante intervención didáctica haya fomentado la motivación del alumnado. Conforme a lo expuesto por la docente en el diario de clase, "el alumnado aprende, repasa o consolida conocimientos sin apenas darse cuenta, ya que el primer objetivo de la actividad para ellos es la diversión y no el aprendizaje. No obstante, aunque el aprendizaje no es el objetivo principal es el resultado más evidente y observado durante la supervisión de estas actividades". Como exponen Gallardo y Gallardo en su análisis de la importancia del juego como herramienta didáctica "el juego es un importante vehículo que tienen los niños para aprender y asimilar nuevos conceptos, 
habilidades y experiencias. Por ello, el juego es una herramienta pedagógica primordial en educación" (2018, p. 49). Asimismo, es preciso resaltar como durante la implementación de juegos como recurso didáctico los estudiantes no solo aprenden los contenidos propios del área, sino también otros de carácter transversal tales como el desarrollo de habilidades sociales, e control emocional, la concepción de normas, etc. (Gallardo y Gallardo,2018).

\section{Conclusiones}

Los resultados del estudio diagnóstico sobre el uso de las distintas estrategias didácticas permiten concluir que los docentes participantes implementan estrategias didácticas en función de su experiencia y su utilidad en el aula y no en función de un modelo didáctico estanco predefinido (objetivo 1). Se observa el énfasis en el uso de estrategias como: el partir de los conocimientos previos, la contextualización de los contenidos, el fomento del dialogo y la reflexión crítica. Asimismo, los resultados del estudio didáctico muestran como el uso de las estrategias didácticas implementadas promueven la motivación del alumnado y con ella un aprendizaje más profundo, reflexivo y contextualizado, acorde con el desarrollo de la alfabetización científica del alumnado (objetivo 2).

Finalmente, se cree preciso destacar el carácter exploratorio del estudio y las limitaciones del mismo, siendo este punto de partida para iniciar una investigación mayor que permita ahondar en las cuestiones aquí destacadas y su posterior interpretación y generalización.

\section{Referencias}

Aubert, A., Flecha, A., García, C., Flecha, R. y Racionero, S. (2010). Aprendizaje Dialógico en la Sociedad de la información. Barcelona: Hipatia.

Bruner, JS. (2000). La educación, la puerta de la cultura. Madrid: Visor.

Cifuentes, A. y Fernández, M. (2010). Proceso de transformación de un centro educativo en Comunidad de Aprendizaje: el Colegio "Apóstol San Pablo" de Burgos (España). Revista Interuniversitaria de Formación del Profesorado, 24(1), 57-73.

De la Hoz, C. (2011). Impacto de las comunidades de aprendizaje en la transformación de las prácticas pedagógicas. Dictamen Libre, 8, 45-48.

Díez-Palomar, J. y Flecha, R. (2010). Comunidades de Aprendizaje: un proyecto de transformación social y educativa. Monográfico sobre Comunidades de Aprendizaje. Revista Interuniversitaria de Formación del Profesorado, 24(1), 19-30.

Dunbar, R. (1999). El miedo a la ciencia. Madrid: Alianza.

Elboj, C., Puigdellívol, I., Soler, M. y Valls, R. (2005). Comunidades de Aprendizaje:Transformar la educación. Barcelona: Grao.

Flecha, R., Prados, M. y Puigdellívol, A. (2003) Comunidades de Aprendizaje: transformar la organización escolar al servicio de la comunidad. Organización y gestión educativa, 5, 4-8.
Freire, P. (1970). Pedagogía del oprimido. Madrid: Siglo XXI.

García-Ruiz, M. y Orozco, L. (2008). Orientando un cambio de actitud hacia las Ciencia Naturales y su enseñanza en Profesores de Educación Primaria. Revista Electrónica de Enseñanza de las Ciencias, 7(3), 539-568.

Gallardo, J. y Gallardo, P. (2018). Teorías sobre el juego y su importancia como recurso educativo para el desarrollo integral infantil. Revista Educativa Hekademos, 24, 41-51.

Garrido, JM., Perales, FJ. y Galdón, M. (2007). Ciencia para educadores. Madrid: Pearson Prentice Hall.

González, E. (2005). Del uso y abuso de los libros de texto: criterios de selección. Revista de educación de la Universidad de Granada, 18, 269-281.

Jiménez, MP. (2000). Modelos didácticos. En J. Perales, y P. Cañal (Ed.), Didáctica de las ciencias experimentales, pp. 170-177. Marfil: Alcoy.

Kvale, S. (2011). Las entrevistas en Investigación Cualitativa. Madrid: Morata.

Lavín, K. (2014). Desarrollo del pensamiento científico por medio de la metodología de grupos interactivos. Revista de Estudios y Experiencias en Educación, 13(26), 67-80. 
Martín, C., Prieto, T., Jiménez, M. A. (2015). Tendencias del profesorado de ciencias en formación inicial sobre las estrategias metodológicas en la enseñanza de las ciencias. Estudio de un caso en Málaga. Enseñanza de las Ciencias, $33(1), 167-184$.

Mosquera, I. (2017). El debate como recurso didáctico innovador. UnirRevista. [WebCite: http://www.webcitation.org/766IVH62l]

Pedrinaci, E. (Coord.), Caamaño, A., Cañal, P., y de Pro, A. (2012). 11 ideas clave. El desarrollo de la competencia científica. Barcelona: Graó

Pujalte, AJ., Bonan, L. y Porro, S. (2014). Las imágenes inadecuadas de ciencia y de científico como foco de la naturaleza de la ciencia: estado del arte y cuestiones pendientes. Ciencia y educación, 20(3), 535-548

Renés, P. (2018). Planteamiento de los estilos de enseñanza desde un enfoque cognitivo-constructivista. Tendencias pedagógicas, 31, 47-67.

Ritchard, R., Church, M., \& Morrison, K. (2011). Making Thinking Visible. How to Promote Engagement Understanding and Independence for All Learners. United States of American: Wiley.

Romero, Y. y Pulido, G. (2015). Incidencia de las Rutinas de Pensamiento en el Fortalecimiento de habilidades Científicas: Observar y Preguntar en los estudiantes de grado cuarto, ciclo II del Colegio Rural José Celestino Mutis I.E.D (Tesis de Posgrado). Universidad de La Sabana, Chía-Cundinamarca.

Solbes, J. (2011). ¿Por qué disminuye el alumnado de ciencias?. Alambique. Didáctica de las Ciencias Experimentales, 67, 5361.

Solbes, J., Montserrat, R. y Furió, C. (2007). El desinterés del alumnado hacia el aprendizaje de la ciencia: implicaciones en su enseñanza. Didáctica de las ciencias experimentales y sociales, 21, 91-117

Taylor, SJ. y Bogdan, R. (2010). Introducción a los métodos cualitativos de investigación. Barcelona: Paidós.

Valls, R. (2005). Los educadores y las educadoras sociales en las Comunidades de Aprendizaje. Revista de Educación Social, 4,5 .

Vázquez, A., Manassero, MA., Acevedo, JA. y Acevedo, P. (2007). Consensos sobre la naturaleza de la Ciencia: la comunidad tecnocientífica. Revista Electrónica de Enseñanza de las Ciencias, 6(2), 331-363.

Vázquez, A. y Manassero, MA. (2011). El descenso de las actitudes hacia la ciencia de chicos y chicas en la educación obligatoria. Ciencia \& Educaçao, 17(2), 249-268.

Vázquez, A. y Manassero, MA. (2012). La selección de contenidos para enseñar naturaleza de la ciencia y tecnología (parte 1): Una revisión de las aportaciones de la investigación didáctica. Revista Eureka sobre Enseñanza y Divulgación de las Ciencias, 9(1), 2-31.

Vílchez, JM. (Coord.), Benarroch, A. Carrillo, FJ., Cervantes, A., Fernández, M. y Perales, F. J. (2015). Didáctica de las ciencias para Educación Primaria I. Ciencias del espacio y de la Tierra. Madrid: Pirámide.
Vygotsky, LS. (1996). El desarrollo de los procesos psicológicos superiores. Barcelona: Crítica.

Zabalza, MA. (2004). Diarios de clase. Un instrumento de investigación y desarrollo profesional. Madrid: Narcea. 